\title{
FORMAÇÃO EM ALTAS CAPACIDADES INTELECTUAIS PARA \\ PROFISSIONAIS PSICOEDUCATIVOS: RESULTADOS DO DESENVOLVIMENTO DO CURSO DA UNIVERSIDADE DE MÁLAGA
}

\author{
LA FORMACIÓN EN ALTAS CAPACIDADES INTELECTUALES DE LOS \\ PROFESIONALES PSICOEDUCATIVOS. RESULTADOS DEL CURSO DE \\ ESPECIALIZACIÓN DE LA UNIVERSIDAD DE MÁLAGA
}

TRAINING IN HIGH INTELLECTUAL CAPACITIES OF PSYCHOEDUCATIONAL PROFESSIONALS. RESULTS OF THE SPECIALIZATION COURSE OF THE UNIVERSITY OF MÁLAGA

\author{
Milagros FERNÁNDEZ-MOLINA ${ }^{1}$
}

RESUMO: O atendimento educacional de alunos com altas habilidades intelectuais necessita da atuação de diferentes profissionais na escola, mas há poucas oportunidades de treinamento disponíveis para eles. Na Universidade de Málaga um curso especializado desde 2011, foi avaliado e desenvolvido para este estudo utilizando o método de pesquisa. Os resultados mostram que há uma alta taxa de inscrição, a maioria mulheres e muitas vezes são estudantes ou graduados em psicologia ou Magistério, com pouca formação ou experiência anterior em alta capacidade. Os alunos fizeram lições muito valiosas sobre o preconceito, intervenção e cooperação com as famílias. Também positivamente eles percebem o currículo aula ministrada e a metodologia utilizada pelos professores. Esses resultados são discutidos ressaltando suas implicações para a melhoria da cultura social sobre a alta capacidade.

PALAVRAS-CHAVE: Formação. Profissionais. Altas habilidades intelectuais. Superdotação.

RESUMEN: La atención educativa de los estudiantes con altas capacidades intelectuales la realizan diferentes profesionales en la escuela, pero son escasas las oportunidades de formación de las que disponen. En la Universidad de Málaga se desarrolla un curso especializado desde 2011, que ha sido evaluado para este trabajo mediante el método de encuesta. Los resultados muestran que existe una tasa de matriculación alta, la mayoría son mujeres y suelen ser estudiantes o graduados en Psicología o Magisterio, con escasa formación ni experiencia previa en alta capacidad. Los alumnos han realizado aprendizajes muy valiosos sobre los prejuicios, la intervención, y la cooperación con las familias. Asimismo se perciben positivamente los contenidos curriculares impartidos yla metodología de clase utilizada por los docentes. Se discuten estos resultados resaltando sus implicaciones de cara a la mejora de la cultura social acerca de la alta capacidad.

${ }^{1}$ Prof ${ }^{a}$ Dra. Do Departamento de Psicología Evolutiva y de la Educación. Facultad de Ciencias de la Educación. Universidad de Málaga Campus Teatinos s/n 29071 Málaga. España - mfernandezm@uma.es 
PALABRAS CLAVE: Formación. Profesionales. Altas capacidades intelectuales. Sobredotación.

ABSTRACT: Gifted students' educational needs are done by different professionals, but there is limited opportunities of formation. The Postgraduate Course on High Abilities is been developed in the University of Malaga from 2011 and has been evaluated in this work by questionaire method. Results show an high enrolment, students are women and they are studying Psychology or Education Sciences and they have not prior experience or studies on gifted children. Students have learnt about prejudices, intervention and collaboration with the families. In addition, they value specially contents and method used by teachers. In this article, we discuss results in relation with improvement of the social perception on gifted children.

KEYWORDS: Training. Education professionals. Teacher education. Gifted children. High abilities. Exceptionally gifted.

\section{Introduccion}

La atención a las necesidades educativas de los estudiantes con altas capacidades intelectuales (ACIs) la realizan diferentes profesionales en el contexto escolar, como maestros, orientadores, profesores, etc., así como psicólogos y terapeutas en contextos más clínicos. En muchos países, la formación universitaria especializada que estos profesionales hayan podido recibir sobre las altas capacidades intelectuales es en la mayoría de los casos deficitaria, debido no sólo a la escasa o residual presencia de estos contenidos en los estudios de licenciaturas o grados, sino también a la falta de estudios de postgrado específicos en ACIs o de cursos de actualización docente.

En España, entre 2008 y 2011, una federación de altas capacidades intelectuales (FASI, 2011), realizó una encuesta pública y abierta en su red, con la pregunta: ¿Consideras que el sistema educativo atiende las necesidades de tu hijo/a?., dirigida especialmente a las familias con hijos ACIs.De los 91 votantes, el 80\% dijo que esta atención era mala o muy mala, el 14\% señaló que regular, y únicamente el 8,8\% manifestó estar satisfecho, calificándola como buena o muy buena. Las reflexiones que suscitan estas respuestas pueden ir encaminadas hacia muchos aspectos del tema, pero sin duda nos hacen descubrir que existe una enorme insatisfacción en la mayoría de los padres y madres sobre cómo la escuela está afrontando las necesidades de estos estudiantes. Posiblemente, la falta de sensibilidad y de formación acerca de las ACIs de 
los profesionales de la escuela que atienden a sus hijos pueda estar en el origen de la pesimista respuesta del $80 \%$ de estas familias.

En otra comunidad autónoma española, la madrileña, el movimiento social contra la Orden1493/2015 sobre necesidades educativas para el alumnado ACIs denunció, mediante una campaña en las redes sociales, la inadecuada atención que se le estaba prestando en esa comunidad al alumnado ACIs. Esta campaña la denominaron "5 formas de hacer desaparecer al alumnado de altas capacidades dentro del sistema educativo madrileño". Los argumentos en los que basaba la queja de este movimiento se centran en varios aspectos que establece la citada Orden y, dentro de ellos, los puntos uno y cincohacen referencia a la capacitación y formación de los profesionales que deben atender a los estudiantes ACIs. Por ejemplo, en el puntouno se establece que "los maestros realizarán la identificación de los alumnos de altas capacidades”. Ante esta afirmación, convertida en orden de obligado cumplimiento en las escuelas,el colectivodenuncia que los maestros que no tienen formación en ACIs no sabrán detectarlos o identificarlos, por lo que muchos alumnos ACIs quedarán ocultos. En el punto cinco se habla de que "la evaluación psicopedagógica la realizará un profesor de la especialidad de orientación educativa”. A este respecto, la ausencia de capacitación profesional en ACIs de estos profesorespuede conllevar errores tanto en la selección de pruebas diagnósticas como en la aplicación de las tareas, y en la corrección de los resultados, como en la aplicación de las medidas educativas que sean pertinentes para cada estudiante derivadas del diagnóstico.

Como hemos comentado al principio, la situación de la formación especializada en ACIs de los profesionales educativos y clínicos ha sido poco alentadora, aunque en los últimos años están surgiendo cada vez más iniciativas, tanto por parte de la universidad como por parte de entidades privadas, que aumentan la oferta formativa especializada en ACIs. La formación en ACIs de los profesionales psicoeducativos, como en cualquier otro contenido que afecte a los niños y niñas con necesidades específicas de apoyo educativo (NEAE), no sólo es imprescindible para que el maestro sepa detectarlos y tratarlos, o el orientador sepa evaluarlos y emitir informes, sino que la formación es vital para detectar y contrarrestar los prejuicios e ideas erróneas sobre las ACIs que pudieran tener estos agentes educativos. Las ideas implícitas sobre la inteligencia y sobre las ACIs existen, influyen en la definición del alumno, en la selección de los contenidos en el aula, en las prácticas educativas concretas de cada profesor, en los mensajes de refuerzo que emita en el aula a cada alumno, en las forma 
de evaluarlos, etc. (Bernardo, 2012; Blackwell, Trzesniewski\& Sorich Dweck, 2012). Unicamente la formación especializada durante años puede actuar como medida de contención de estas ideas implícitas inadecuadas (García Bacete \& Rosel, 1999).

Ahora bien,no sólo es necesario e importante diseñar actividades formativas para los profesionales que trabajan con los alumnos ACIs, evaluar los programas que se dedican a formar a esos profesionales es también prioritario.Es importante conocer todo lo relacionado con las acciones formativas que implementemos porque, en primer lugar,nos va a permitir diseñar y proporcionar ofertas formativas más atractivas que convenzan a los posibles alumnos, frente a otros contenidos tradicionalmente más demandados como la formación en atención temprana, en aprendizaje cooperativo, en dificultades de aprendizaje, etc. En segundo lugar, permite aumentar el número de profesionales sensibilizados y formados en ACIsde manera seria y rigurosa, y por último, posibilitadetectar los puntos fuertes y débiles de nuestra oferta formativa para su mejora, ampliación o, si fuese el caso, su desaparición.

El Curso de Especialización en ACIs de la Universidad de Málaga (CEACIsUMA) es un título propio en ACIs (Fernández-Molina, 2016). La formación de postgrado de las universidades españolas se ha reconvertido desde el proceso de convergencia al Espacio Europeo de Enseñanza Superior. La estructura de esta formación ha quedado establecida en Máster, Expertos y Titulaciones Propias o de Especialización, que a su vez pueden ser para alumnado de últimos años, llamados Cursos de Extensión Universitaria, o bien para alumnado ya graduado o profesionales externos, denominados Cursos de Especialización.

El CEACIs-UMA se ha venido desarrollando durante 5 ediciones consecutivas entre los cursos académicos 2011-2012 y 2015-2016, y se vuelve a ofertar de cara al curso 2016-2017. La oferta formativa consiste en 6 créditos ECTS, lo que significa 60 horas de clases, tanto presenciales como virtuales, con una exigencia total de 150 horas de trabajo de los alumnos. Esto supone que la diferencia de horas entre las 60 de clases presenciales y virtuales y las 150 horas de trabajo total, deben ser invertidas por el alumnado en aquellas actividades establecidas por el profesorado que requieren trabajo autónomo como, por ejemplo, lectura comprensiva de los materiales, subrayado y resumen de los contenidos, estudio privado, visionado de películas o vídeos, búsqueda de materiales, redacción de trabajos, cumplimentación de encuestas o test de evaluación, etc. La estructura semipresencial de la docencia de este curso se divide así: las seis clases presenciales se imparten los sábados en horario de 9 a 14 o de 10 a 14 horas, 
distribuidos entre octubre y febrero; las clases virtuales se imparten desde la plataforma moodle de la propia Universidad de Málaga. Los contenidos del curso se distribuyen en siete temas que tienen todos una parte presencial y una parte virtual. Los contenidos del CEACIs-UMA se muestran en la Tabla 1.

Tabla 1. Competencias, contenidos y actividades del CEACIs-UMA (20112016)

\section{Competencias \\ Conocer los modelos actuales sobre inteligencia humana y su relación con la práctica educativa, superando las ideas implícitas erróneas sobre las altas capacidades intelectuales.}

Conocer y valorar la diversidad del alumnado con altas capacidad intelectuales

Contenidos
INTELIGENCIA, ALTAS
CAPACIDADES Y
ESCUELA

DIVERSIDAD DEL ALUMNADO CON NEAE POR ACIS

\section{NORMATIVA VIGENTE \\ NACIONAL Y \\ AUTONOMICA}

hacia el alumnado con altas

capacidades intelectuales

Saber utilizar los criterios y procedimientos establecidos para la detección e identificación de este alumnado.

Conocer y analizar las necesidades educativas de este alumnado

Ser capaces de tomar decisiones adecuadas sobre medidas y orientaciones psicoeducativas

\section{NECESIDADES \\ EDUCATIVAS ESPECIFICAS DEL ALUMNADO CON ACIS}

BAJO RENDIMIENTO ESCOLAR EN EL ALUMNADO ACIS

\section{Actividades}

Seminarios presenciales de debate, reflexión y análisis.

Lectura de material multimedia Prácticas sobre Modelos de inteligencia, Ideas implícitas, Cuestionarios de detección, etc. Realización de esquemas o mapas conceptuales

Realización de encuestas de autoevaluación del proceso de aprendizaje

Seminarios presenciales de debate, reflexión y análisis.

Lectura de material multimedia Prácticas sobre Modelos de inteligencia, Ideas implícitas, Cuestionarios de detección, etc. Visionado y reflexión crítica de videos sobre la realidad del alumnado con altas capacidades Realización de encuestas de autoevaluación del proceso de aprendizaje

Búsqueda en la legislación vigente por objetivos

Realización de encuestas de autoevaluación del proceso de aprendizaje

Seminarios presenciales de debate, reflexión y análisis.

Lectura de material multimedia

Prácticas sobre Modelos de inteligencia, Ideas implícitas, Cuestionarios de detección, etc. Realización de encuestas de autoevaluación del proceso de aprendizaje

Seminarios presenciales de debate, reflexión y análisis

Lectura de material multimedia Búsqueda en la legislación vigente por objetivos

Visionado y reflexión crítica de videos sobre la realidad del alumnado con altas capacidades Estudio de casos Estudio y análisis de instrumentos 
Iniciarse en la metodología de intervención básica para el enriquecimiento curricular y extracurricular de este alumnado a través del conocimiento de la mediación del aprendizaje y programas o prácticas educativas eficaces

Desarrollar actitudes sensibles hacia la imprescindible relación cooperativa con las familias, conociendo los recursos del asociacionismo familiar en altas capacidades intelectuales, así como otros recursos de colaboración familia-escuela y procedimientos de intervención

Realización de encuestas de autoevaluación del proceso de aprendizaje

BUENAS PRACTICAS EN Seminarios presenciales de debate, EL AULA. ESTRATEGIAS reflexión y análisis.

Y RECURSOS DE

ENRIQUECIMIENTO

Lectura de material multimedia

Estudio de casos

Estudio y análisis de instrumentos y procedimientos de intervención

Realización de encuestas de autoevaluación del proceso de aprendizaje

FAMILIA-ESCUELA Y Seminarios presenciales de debate, ACIs reflexión y análisis.

Lectura de material multimedia Elección e investigación sobre las actividades de enriquecimiento del asociacionismo familiar

Estudio de casos

Estudio y análisis de instrumentos y procedimientos de intervención Realización de encuestas de autoevaluación del proceso de aprendizaje

Fonte: elaborada pela autora

Los destinatarios del curso han sido titulados en Psicopedagogía, Psicología o Pedagogía; trabajadores y profesionales del sector educativo público, privado y concertado, tanto tutores/as como jefes/as de estudio, coordinadores/as de ciclo, directores/as, etc., tanto en activo como en situación de desempleo; padres y madres con hijos/as ACIs; estudiantes del Grado de Psicología, Maestro/a en Educación Primaria, Maestro/a en Educación Infantil, Pedagogía, Educación Social.; estudiantes o titulado/as de Cursos de Postgrado relacionados con la Intervención Psicoeducativa, como el Máster de Profesorado en Educación Secundaria Obligatoria y Bachillerato, Formación profesional y Enseñanzas de Idiomas; Máster en Orientación e Intervención Psicoeducativa, Máster en Políticas y Prácticas Educativas, Máster en Psicopedagogía, etc.; estudiantes matriculados/as en asignaturas de los Planes a extinguir de las titulaciones de: Maestro/a Audición y Lenguaje, Maestro/a Educación Especial, Maestra/o Educación Física, Maestro/a Educación Infantil, Maestra/o Educación Musical, Maestra/o Educación Primaria, Maestro/a Lengua Extranjera, Educación Social. Psicología, Pedagogía, Psicopedagogía.

\section{Objetivos}


Los objetivos de este trabajo son en primer lugar, conocer el perfil de los profesionales y futuros profesionales que se han interesado por formarse en ACIs; en segundo lugar, definir los procesos de aprendizaje que han realizado los participantes en el CEACIs-UMA; y en tercer lugar, conocer la percepción y la valoración que hacen los participantes del propio CEACIs-UMA, para de esta forma poder establecer medidas de mejora para futuras ediciones.

\section{Metodo}

\section{Participantes}

Como participantes en el estudio se tomaron a todos los alumnos matriculados en las cinco ediciones del curso objeto de este trabajo, aunque finalmente se incluyeron como participantes los de las tres últimas ediciones, excluyendo tanto a los de la primera edición (2011-2012) como a los de la última (2015-2016). Las respuestas de los alumnos de la primera edición debieron ser anuladas debido a problemas técnicos con el registro de sus respuestas en la plataforma virtual. Los alumnos de la ultima edición no fueron incluidos en este estudio debido a que en el momento de realizar este trabajo no se había terminado de recoger los datos de esta promoción.

En la Tabla 2 se presentan los datos relativos al número de alumnos preinscritos, número de alumnos efectivamente matriculados y número de alumnos que, de entre los matriculados, finalmente respondieron a este estudio. El número de plazas ofertadas fue entre 15 y 40. Como se observa en la tabla el interés por el curso fue muy alto superando el $100 \%$ de las plazas ofertadas.Más del 60\% de los alumnos interesados en el curso formalizaron finalmente la matrícula llegando a tasas de matriculación del 84\%, e incluso en el curso 2013-2014 hubo que aumentar el número máximo de matrículas para atender la demanda. Como también se muestra en la tabla 2, la tasa de participación en el estudio fue muy alta, encontrando una tasa de respuesta por encima del $75 \%$ de los matriculados. La mayoría de los participantes fueron mujeres, siendo el porcentaje de mujeres del $87,5 \%$ en el curso $2011-2012$, del $87,5 \%$ en el curso 2012 2013, del 88,4\% en el 2013-2014 y, del 89\% en el último curso. 
Tabla 2. Distribución de alumnos de las cinco ediciones del CEACIs-UMA

\begin{tabular}{lllllll}
\hline & \multicolumn{2}{c}{ Preinscritos } & \multicolumn{2}{c}{ Matriculados } & \multicolumn{2}{l}{ Respondientes } \\
& $\mathrm{N}$ & $\%$ & $\mathrm{~N}$ & $\%$ & $\mathrm{~N}$ & $\%$ \\
Curso 2011-2012 & 48 & $120 \%$ & 40 & $83 \%$ & 34 & $85 \%$ \\
Curso 2012-2013 & 54 & $135 \%$ & 40 & $74 \%$ & 32 & $80 \%$ \\
Curso 2013-2014 & 51 & $127,5 \%$ & 43 & $84 \%$ & 34 & $79 \%$ \\
Curso 2014-2015 & 41 & $102,5 \%$ & 26 & $63 \%$ & 26 & $100 \%$ \\
Curso 2015-2016 & 22 & $55 \%$ & 18 & $82 \%$ & 0 & $0 \%$ \\
\hline
\end{tabular}

Fonte: elaborado pela autora

\section{Procedimiento}

La recogida de datos se realizó en cuatro puntos temporales a lo largo de la realización del curso: al principio del curso, al terminar los temas 1-2-3; al finalizar los temas 4-5; y por último al acabar los temas 6-7. Se evaluaron tanto las sesiones presenciales como las virtuales, las actividades programadas para el aprendizaje, los seis docentes que impartieron clases en el curso y el grado de valoración general del curso. La tasa de asistencia al curso fue superior al 90\% en los temas 1,2,3 y en los temas 6 y 7 para las tres ediciones del curso analizadas. Para los temas 4 y 5 fue superior al $80 \%$ para las dos primeras ediciones y del $60 \%$ en la tercera edición del curso analizada. Se utilizó análisis de descriptivos mediante excell y análisis de contenido de las respuestas cualitativas.

\section{Instrumentos}

La evaluación se realizó a través de la misma plataforma virtual en la que se impartía la parte virtual del curso pero que servía de espacio tanto de docencia como de comunicación entre docentes y alumnado. Se utilizó el método de encuestas elaborando un Cuestionario con varios apartados que recogían la información necesaria para el estudio. En la tabla 3 se muestran las preguntas utilizadas.

Tabla 3. Encuesta utilizada para la recogida de datos 
Procesos de aprendizaje que han hecho durante el curso: ideas previas, conocimientos previos sobre ACIs, aprendizajes básicos sobre ACIs, medidas educativas para ACIs, buenas prácticas en los diferentes niveles escolares

Percepción y valoración de curso qué titulación tienes y/o qué estudios estás cursando en la actualidad

Experiencia previa en altas capacidades: Tienes experiencia en el ámbito de las ACIS. Explica cuál es tu experiencia concreta. Has recibido alguna otra formación en ACI. Explícanos qué otra formación has recibido y por parte de qué organismo o entidad

Expectativas sobre el curso: Explícanos qué esperas encontrar en este TP, cuál era tu principal objetivo cuando te matriculaste de este curso Resume, brevemente, sin mirar el material ni las actividades del curso, lo que has aprendido sobre ACIS hasta el momento. Lo que he aprendido es que...

Si cierras los ojos y sin hacer esfuerzo recuerdas todos los contenidos, actividades, mensajes, materiales recursos, técnicas, etc. que te hemos ido mostrando durante TODO EL CURSO, lo que te viene a la mente es...Lo que crees que has aprendido en este curso es que...

De las sesiones presenciales a las que has asistido hasta el momento, ¿cuál de ellas te ha resultado más fructífera, más interesante? Puedes marcar hasta 3 opciones.

¿Cuáles son los aspectos que más te han gustado de las sesiones presenciales? Puedes comentar sobre organización, contenidos, materiales, etc. Cómo valoras a cada docente en una escala de 1 a 5 puntos: 1 Muy mal, 2 Mal, 3 Normal, 4 Bien, 5 Muy bien.

Resume en una frase el aspecto que más valoras de TODO el curso. Lo que más me ha gustado ha sido...

Resumen en una frase el aspecto que menos te ha gustado de TODO el curso. Lo que menos me ha gustado ha sido...

Fonte: elaborada pela autora

\section{Resultados}

\subsection{Perfil del alumnado participante}

Los resultados acerca del perfil del alumnado del curso se refieren a las titulaciones previas que tenían antes de hacer el curso, las motivaciones para elegir esta formación en lugar de otras disponibles en su entorno, y la experiencia y la formación previa en ACIs.

El perfil de los participantes en el estudio muestra que la mayoría proceden de los estudios de Grado de Magisterio y de Psicología. Así, en la edición de 2012-2013, el 
44\% estaba cursando o tenían estudios de Psicología o Pedagogía, y en las ediciones de 2013-2014 y 2014-2015, el 35\% y el 45\%, respectivamente, tenían estudios de graduados en Maestro de Educación Primaria o Infantil.

Los porcentajes de respuesta muestran que los participantes decidieron realizar el curso especialmente atraídos por los contenidos del mismo. El 67\% en el curso 20122013, el 82\% en el curso 2013-2014, y el 75\% en el curso 2014-2015, decidieron elegir este título de especialización gracias a su oferta curricular. El otro aspecto mencionado fue la combinación de clases presenciales y virtuales, para el 22\% en el curso 20122013, para el 9\% en el 2013-204 y para el 6\%, en el 2014-2015. En cambio, el profesorado del curso no fue un elemento valorado a la hora de seleccionar el curso por casi ningún participante. Otros elementos que pudieran atraer hacia el curso, como el precio, la flexibilidad horaria o el emitir un certificado oficial fueron motivos atrayentes para el 11\%, en el curso 2012-2013, para el 6\% de los participantes en el 2013-2014, y para el 18\% en el año 2014-2015.

En cuanto a las expectativas sobre la formación que les iba a proporcionar el curso, los alumnos señalaron que su principal motivación era aprender para poder intervenir con estos niños, especializándose en la atención del alumnado ACIs y poder trabajar en gabinetes propios o ajenos, o en colegios $(64 \%, 48 \%$ y $78 \%$, respectivamente). La otra gran motivación eraconocer sobre las ACIs y actualizar sus conocimientos $(25 \%, 35 \%$ y $45 \%$, respectivamente).

En la tabla 4 se muestran los resultados en cuanto a la experiencia previa y en formación previa en ACIs que tenían los alumnos. Como muestran los datos, la mayoría declaró no tener ninguna formación ni experiencia previa acerca de las altas capacidades. Unicamente en la última edición del curso se encuentran casi los mismos porcentajes de participantes con y sin formación y experiencia previa. Los alumnos que dijeron tener formación previa explicaron que era debido a que habían realizado otros cursos en entidades privadas o asociaciones vinculadas a las altas capacidades, o a que eran madre y padres con hijos ACIs y por ello se habían preocupado por formarse en ACIs de forma autodidacta.

Tabla 4. Formación y experiencia previa en ACIs de los participantes

\begin{tabular}{|c|c|c|c|c|}
\hline & \multicolumn{2}{|c|}{ Formación previa en ACIs } & \multicolumn{2}{c|}{ Experiencia previa en ACIs } \\
\hline & SI & NO & SI & No \\
\hline $2012-2013$ & $30 \%$ & $70 \%$ & $15 \%$ & $85 \%$ \\
\hline $2013-2014$ & $38 \%$ & $62 \%$ & $35 \%$ & $65 \%$ \\
\hline
\end{tabular}




\begin{tabular}{|l|l|l|l|l|}
\hline $2014-2015$ & $50 \%$ & $50 \%$ & $44 \%$ & $56 \%$ \\
\hline
\end{tabular}

Fonte: elaborado pela auotra

\subsection{Procesos de aprendizaje que han realizado gracias al curso}

Según los datos obtenidos, los aprendizajes que los alumnos participantes en el estudio pudieron adquirir estaban relacionados principalmente con estos contenidos: a) ideas implícitas y prejuicios acerca de las ACIs, b) definición o concepto de altas capacidad y de alumnado ACIs, c) trabajo en la escuela con esta población, detección de las ACIs, intervención en el aula y por último, d) interacción con las familias.

En relación a los prejuicios, los alumnos aprendieron que estas ideas previas existen, las puedenconstruir y tener ellos mismos, que deben estar alerta para detectarlas sobre todo si son docentes, trabajar conscientemente para desmontarlas, y además que estos prejuicios reflejan un gran desconocimiento sobre las ACIs. Asimismo, sus repuestas denotan un aumento de la sensibilidad sobre este tipo de alumnado, ya que han aprendido que deben ponerse en el lugar de todos los implicados: padres, hijos, hermanos, tutores, etc. Algunas de las respuestas cualitativas que reflejan estos aprendizajes son:

"Me he hecho consciente de que tenía muchos de mis conocimientos sobre altas capacidades marcados por mitos y estereotipos" (Participante.2, 2012-2013)

"Es necesario desmitificar y crear una conciencia colectiva respecto al tema lo que llevará a aportar a los niños una educación adecuada y justa" (P.4, 2013-2014)

"No tenía más que conocimientos que los propios marcados por mitos, los que he eliminado y borrado para sustituirlos por un enorme arsenal de contenido tanto teórico como en su puesta en práctica" (P.22, 2014-2015)

Sobre el concepto o la definición de lo que es la alta capacidad y cómo deben concebirse el alumnado ACIs, las respuestas reflejan que estosparticipantes han aprendido fundamentalmente que la alta capacidad esuna característica más de la persona, es un niño más, un alumno más. Señalan que han aprendido que es necesario conocerlos, así como la complejidad y diversidad de este alumnado. Han aprendido que existen tipos de perfiles y por tanto a diferenciar superdotación, talentos, precocidad, genio, etc. Otro aspecto adquirido ha sido la visión global de las ACIs, entendiéndolas desde lo escolar pero también desde la parte legal, familiar, social y afectiva. Asimismo, los participantes comentan en sus respuestas acerca del desconocimiento de la sociedad sobre esta temática, pero a la vez se dan cuenta que está cambiando la visión sobre las 
ACIs y son optimistas al respecto. Algunas de las respuestas que reflejan estos aprendizaje son:

\begin{abstract}
"La diferencia entre superdotado, talento simple y talento complejo, precoz y genio"(Participante 7, 2012-2013)

"Un mundo nuevo para mí, interesantísimo, donde no conocía absolutamente nada d que existían tanta variedad de niños con altas capacidades"(Participante 9, 20122013)

"Son niños. Hay que tratar la alta capacidad como una característica más" (Participante 14, 2012-2013)
\end{abstract}

Los contenidos que los participantes han adquirido, gracias al curso, acerca del trabajo en la escuela se refieren principalmente a hacerse conscientes de que un día pueden encontrase con alumnado ACIs en las aulas, al proceso para trabajar, incluyendo la detección, y a la metodología de trabajo que deben seguir, es decir, a la intervención, a la diversidad de cosas que pueden hacer para fomentar el desarrollo cognitivo, personal y social de estos niños. Por ejemplo, algunos participantes decían:

\footnotetext{
"Necesidad de motivar tanto al profesorado como a la familia para que entiendan la importancia de la detección temprana y una intervención ajustada a las necesidades de cada niño" (Participante 7, 2012-2013)

"Todo alumnado con o sin altas capacidades necesita despertar su implicación. Llegar a su motivación por el aprendizaje a través de la metodología adecuada. Es la escuela quien debe adaptarse a las características y a las formas de aprendizaje. (Participante 11, 2013-2014)
}

En concreto, en relación a las funciones relacionadas con la detección, los participantes han aprendido acerca de losindicadores en el aula que pueden observar, que una mala conducta puede esconder una alta capacidad así como las diferentes herramientas que tienen a su disposición para detectar (como los cuestionarios de screening adaptados a diferentes edades). Por último han aprendido cómo transmitir esa información a las familias.

\footnotetext{
"El diagnóstico de altas capacidades es muy serio y a su vez importante tratarlo con cautela"(Participante 3, 2012-2013)

"Es muy importante detectar lo antes posible las altas capacidades y trabajar con ellos"(Participante 15, 2014-2015)
}

Sobre la intervención, señalan que los estudiantes ACIs tienen NEAE y que, por tanto, hay que atenderlos. Para ello han asimilado que la escuela debe cambiar y han adquirido metodología adecuada en el aula para ayudarles, ofreciéndoles lo que 
necesitan: motivación, interés, diversión, personalización de la respuesta educativa, elección de actividades, recursos y materiales. Se han hecho conscientes de la importancia de la mediación, como el papel principal del docente y son capaces de señalar algunas pautas para el desarrollo académico, afectivo y social de su alumnado ACIs.

"Existen medidas que se pueden adoptar en el ámbito de la escuela que aunque no se pongan en práctica con la asiduidad que se debiera" (Participante 7, 2012-2013)

"Actividades de ampliación, innovadoras, conociendo antes sus intereses, sus puntos fuertes y sus aficiones" (Participante 3, 2014-2015)

Por último, respecto a la relación o el trato con las familias, el principal aprendizaje que hemos encontrado se refiere a entender a las familias. Los participantes dicen haber aprendido sobre todo a darse cuenta de que existen puntos de vista diferentes al del profesorado, como es el de las familias, y a que deben colaborar con ellas. Por otro lado, han aprendido que existen asociaciones que sirven de red de apoyo y que las familias deben atender las necesidades sociales y emocionales de sus hijos.

"Hacer partícipe a la familia de la detección"(Participante 18, 2012-2013)

"A ver puntos de vista diferentes al de la profesión, como el punto de vista de los padres" (Participante 6, 2013-2014)

"La necesidad de una adecuada coordinación familia-escuela a la hora de ajustar la respuesta educativa para estos alumnos y satisfacer sus necesidades educativas" (Participante 1, 2014-2015)

\subsection{Percepción y valoración del curso}

Se recogió la opinión de los estudiantes acerca del curso, registrando los datos relativos al interés de las sesiones presenciales y virtuales, es decir, recogiendo datos sobre los aspectos que los participantes consideraron más fructíferos para su formación.

En la Tabla 5 se presentan los resultados relativos a la valoración media de las sesiones presenciales según la opinión de los participantes. Como se muestra la mayoría de los porcentajes se sitúan en valoraciones positivas o muy positivas con excepción de la sesión cuarta, dedicada a la legislación, que ha sido la peor valorada. Por otro lado se observa que la tasa de asistencia va decayendo a medida que avanzan las sesiones, de 
forma que hay un $4 \%$ que no asiste a la quinta sesión y sube al $7 \%$ de participantes que reconocen no haber asistido a la última sesión

Tabla 5. Valoración media de las sesiones presenciales, según los alumnos participantes

\begin{tabular}{|l|l|l|l|l|l|l|}
\hline & Sesión 1 & \multicolumn{1}{|c|}{ Sesión 2 } & Sesión 3 & \multicolumn{1}{|c|}{ Sesión 4 } & \multicolumn{1}{|c|}{ Sesión 5 } & \multicolumn{1}{|c|}{ Sesión 6 } \\
\hline Muy mal & $0 \%$ & $0 \%$ & $0 \%$ & 0 & $3,6 \%$ & 0 \\
\hline Mal & $0 \%$ & $0 \%$ & $0 \%$ & $9,3 \%$ & $19 \%$ & 0 \\
\hline Normal & $20,6 \%$ & $9 \%$ & $10 \%$ & $16,6 \%$ & $42 \%$ & $7,6 \%$ \\
\hline Bien & $31,3 \%$ & $35,6 \%$ & $19,3 \%$ & $26 \%$ & $20,6 \%$ & $23 \%$ \\
\hline Muy bien & $37,6 \%$ & $59,6 \%$ & $57,3 \%$ & $41,6 \%$ & $10 \%$ & $61 \%$ \\
\hline No asistí & $0 \%$ & $0 \%$ & $3 \%$ & $3 \%$ & $4 \%$ & $7,6 \%$ \\
\hline
\end{tabular}

Fonte: elaborado pela autora

Para detallar un poco más la valoración de las sesiones, analizamos la percepción de los alumnos acerca de cada uno de los docentes del curso, cinco en total., en las tres ediciones analizadas. Para no extendernos, mostraremos únicamente los resultados obtenidos por el docente más valorado y por el docente menos valorado, según la puntuación media alcanzada en cada edición del curso. En la Figura 1 se muestra la distribución de respuestas del docente que obtuvo la valoración media más alta, y en la figura 2 la distribución de respuestas para el docente peor valorado.

Figura 1. Docente mejor valorado del curso. Perfil de respuestas durante las tres ediciones analizadas.

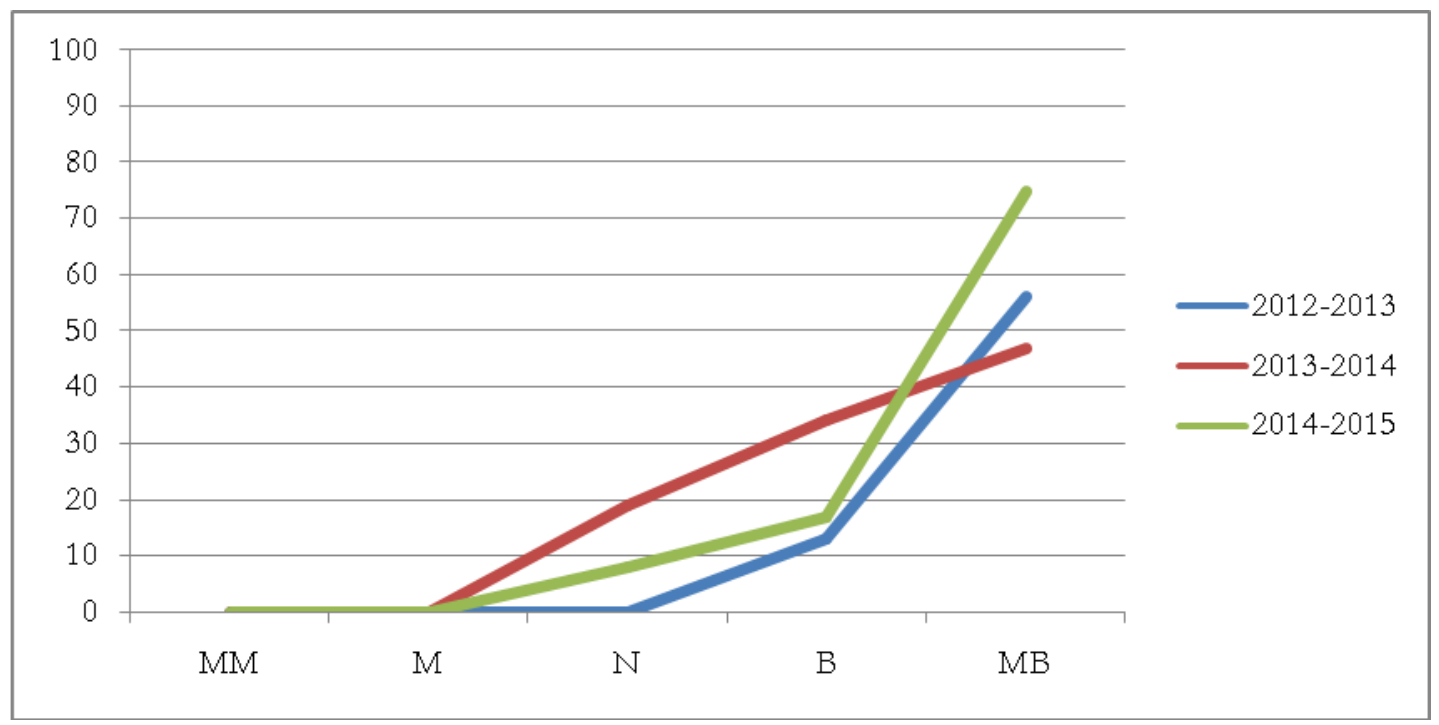


Figura 2. Docente peor valorado del curso. Perfil de respuestas durante las tres ediciones analizadas.

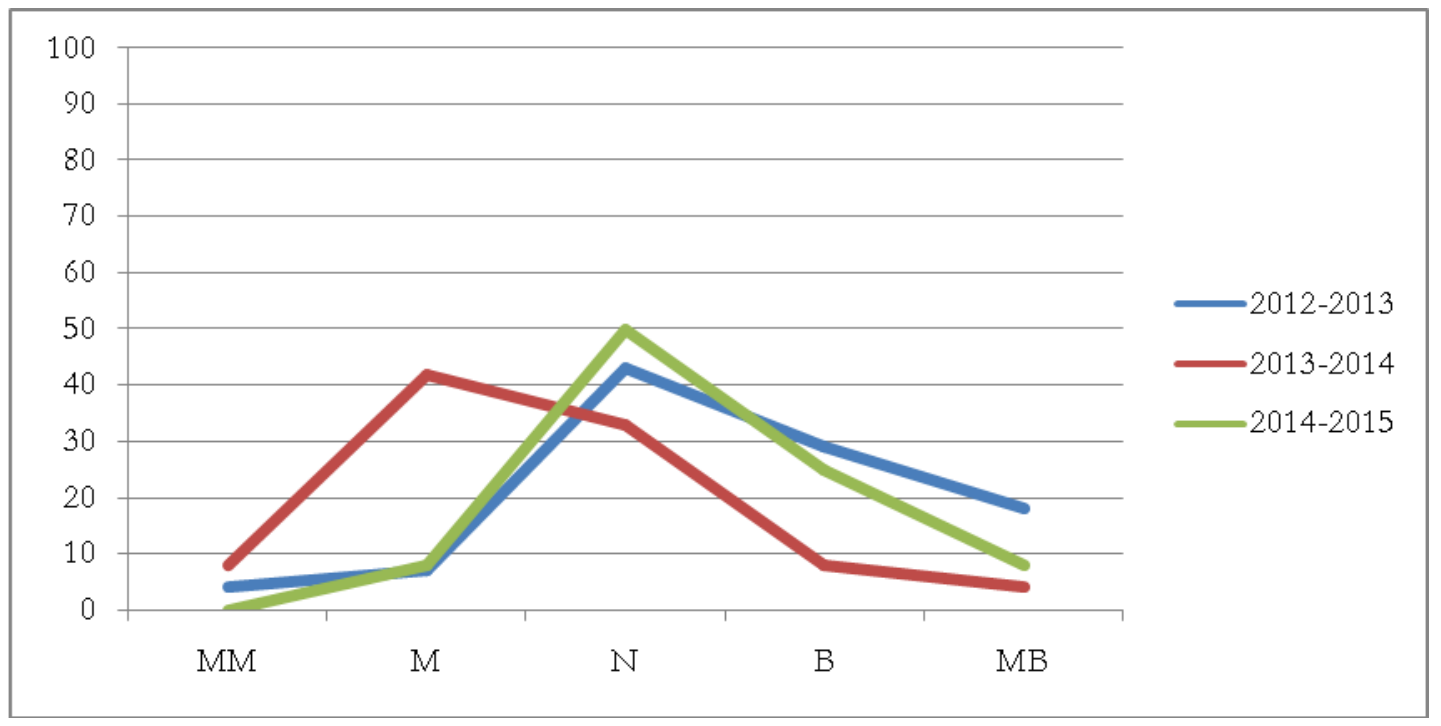

En la Tabla 6 se muestran los resultados acerca de la valoración que hicieron los alumnos participantes sobre las sesiones presenciales del curso. En concreto, se muestran los datos obtenidos sobre los aspectos mejor valorados.

De los cuatro aspectos valorados los porcentajes medios de satisfacción más altos se concentraron en la dimensión relativa a la metodología docente seguida en el curso. Así, todos los participantes destacaron el clima de clase creado gracias a usar el aprendizaje cooperativo, la oportunidad de realizar actividades en pequeños grupos, como debates, análisis de casos, propuestas de intervención etc. Más de la mitad valoraron positivamente la cercanía en el trato y la empatía desplegada por los docentes del curso. Muchos alumnos también valoraron que el curso tuviera contenidos teóricos afianzados mediante actividades de tipo práctico.

Otros aspectos bien valorados por bastantes alumnos fueron el cumplimiento de los horarios y de la organización y planificación del curso $(42,3 \%)$ o los contenidos trabajados acerca de las nuevas teorías sobre inteligencia y modelos explicativos sobre la alta capacidad $(31 \%)$.

Tabla 6. Sesiones presenciales: porcentaje medio de los aspectos mejor valorados

\begin{tabular}{llc} 
DIMENSIONES & $\mathrm{N}$ \\
CONTENIDOS & Características de los niños/as & $15,4 \%$ \\
\hline
\end{tabular}




\begin{tabular}{|c|c|c|}
\hline & Falsos mitos & $23 \%$ \\
\hline & Variabilidad/diversidad de las altas capacidades & $3,8 \%$ \\
\hline & Nuevas Teorías y Modelos & $31 \%$ \\
\hline & NEAE por AACC & $3,8 \%$ \\
\hline RECURSOS & Audiovisuales & $26,9 \%$ \\
\hline & Enorme variedad y cantidad de documentación & $3,8 \%$ \\
\hline & Presentaciones en clase de los profesores & $7,7 \%$ \\
\hline & Ejemplos para detectar y observar & $11,5 \%$ \\
\hline & Diversas respuestas educativas & $7,7 \%$ \\
\hline METODO & Estructuración y orden, contenidos graduados & $19,2 \%$ \\
\hline & Combinación Teoría-Práctica & $46,1 \%$ \\
\hline & Casos reales & $23 \%$ \\
\hline & Tareas grupales, espacios para conocerse y debatir & $100 \%$ \\
\hline & Cercanía en el trato, forma de transmitir conocimientos & $57,7 \%$ \\
\hline ORGANIZACION & Cumplimiento con Aula, horario y planificación estable & $42,3 \%$ \\
\hline & Selección, orden de contenidos, explicación de detalles & $15,4 \%$ \\
\hline & Contar con una plataforma virtual para hacer o colgar ejercicios & $23 \%$ \\
\hline
\end{tabular}

Elaborado pela autora

En el caso de la parte virtual del curso, las actividades mejor valoradas por la mayoría de los participantes fueron: el videoforum (53,5\%), la actividad sobre metodología para alumnado ACIs $(49,3 \%)$, la tarea sobre análisis de los procesos cognitivos implicados en una tarea escolar (47\%), la tarea sobre cómo mediar cognitivamente (46\%), o la tarea de las ideas implícitas $(44,5 \%)$ sobre alta capacidad.

Para finalizar se recogió la valoración global, tanto positiva "lo mejor del curso ha sido..." como negativa "lo peor del curso ha sido...", que hicieron los participantes. Como se muestra en la Tabla 7, los alumnos vuelven a recordar la metodología docente como el aspecto mejor considerado para dos de las tres promociones. Es decir, los debates, el ambiente y clima del aula, las experiencias narradas por los docentes o por los propios alumnos, el material y la información para consulta que los profesores ponían al servicio de los alumnos.El otro aspecto bien valorado han sido los aprendizajes realizados especialmente aquellos referidos al concepto de alta capacidad y a la forma de abordar las ACIs en el aula. 
En cambio, entre los elementos peor valorados los participantes de las tres promociones analizadas recuerdan el tema de la legislación específica en ACIs, También existen algunas valoraciones negativas debido a los contenidos o las tareas, sobre todo en el curso 2013-2014 o sobre la duración de la sesiones presenciales también en el curso 2013-2014. Otros aspectos considerados negativamente por algunos alumnos tenían que ver con el precio del curso, o tener que desplazarse para asistir a las sesiones presenciales.

Tabla 7. Valoración final general del curso. Lo mejor y lo peor valorado. Dimensiones, frecuencia de respuesta

\begin{tabular}{|c|c|c|c|c|c|c|c|}
\hline \multicolumn{4}{|c|}{ Lo mejor del curso ha sido... } & \multicolumn{4}{|c|}{ Lo peor del curso ha sido... } \\
\hline \multirow[t]{2}{*}{ Dimensiones } & \multicolumn{3}{|l|}{$\mathrm{N}$} & Dimensiones & \multicolumn{3}{|c|}{$\mathrm{N}$} \\
\hline & $\begin{array}{l}2012- \\
2013 \\
\mathrm{~N}=21 \\
(52,5 \%)\end{array}$ & $\begin{array}{l}2013- \\
2014 \\
N=\quad 33 \\
(76,7 \%)\end{array}$ & $\begin{array}{l}2014- \\
2015 \\
\mathrm{~N}=16 \\
(61,5 \%)\end{array}$ & & $\begin{array}{l}2012- \\
2013 \\
\mathrm{~N}=\quad 21 \\
(52,5 \%)\end{array}$ & $\begin{array}{l}2013- \\
2014 \\
N=\quad 33 \\
(76,7 \%)\end{array}$ & $\begin{array}{l}2014- \\
2015 \\
\mathrm{~N}=16 \\
(61,5 \%)\end{array}$ \\
\hline $\begin{array}{l}\text { Aprendizajes } \\
\text { realizados }\end{array}$ & 13 & 10 & 4 & $\begin{array}{l}\text { Quejas sobre } \\
\text { contenidos o } \\
\text { tareas }\end{array}$ & 5 & 8 & 2 \\
\hline $\begin{array}{l}\text { Metodología } \\
\text { de clases }\end{array}$ & 6 & 20 & 14 & $\begin{array}{l}\text { Carga } \mathrm{de} \\
\text { trabajo }\end{array}$ & 1 & 4 & 2 \\
\hline Profesorado & 2 & 3 & 2 & $\begin{array}{l}\text { Tema } \\
\text { legislativo }\end{array}$ & 6 & 11 & 5 \\
\hline $\begin{array}{l}\text { Entrega de } \\
\text { tareas }\end{array}$ & 4 & 2 & 0 & $\begin{array}{l}\text { Duración de } \\
\text { las sesiones }\end{array}$ & 4 & 7 & 2 \\
\hline & & & & $\begin{array}{l}\text { Dinámicas } \\
\text { poco } \\
\text { provechosas }\end{array}$ & 3 & 3 & 2 \\
\hline & & & & $\begin{array}{l}\text { Otros } \\
\text { aspectos }\end{array}$ & 2 & 3 & 2 \\
\hline
\end{tabular}

Fonte: elaborada pela autora

\section{Conclusiones}


El Curso de Especialización en altas capacidades intelectuales de la Universidad de Málaga (España) es un título propio que viene ofertándose con bastante éxito desde el año 2011-2012 hasta la actualidad, formando ya a 167 profesionales psicoeducativos. Analizar las características de cursos como éste es vital, no sólo por su posible efecto en las ideas implícitas y en las competencias que tienen estos profesionales en la detección e intervención con el alumnado ACIs, sino también por la necesidad imperiosa de que cualquier actuación de este tipo en el campo de las ACIs sea sometida a procesos de evaluación de su calidad.

Los objetivos de este trabajo han sido conocer el perfil de estos profesionales y futuros profesionales, que se han interesado por formarse en ACIs, definir los procesos de aprendizaje que han realizado y conocer la percepción y la valoración que hacen del curso, para de esta forma poder establecer medidas de mejora para futuras ediciones.

Los resultados acerca del perfil de estos profesionales o futuros profesionales que se interesaron en formarse en ACIs muestran que la mayoría son mujeres, proceden de los estudios de Grado de Magisterio y de Psicología, se decidieron a realizar el curso especialmente atraídos por la propia oferta curricular y por la combinación de clases presenciales y virtuales, no tenían formación ni experiencia previa sobre ACIs y su interés principal era aprender para poder intervenir con estos niños, considerando el curso como un medio necesario con el que poder especializarse en la atención del alumnado ACIs y poder trabajar bien en escuelas o en gabinetes privados de psicología. Estos resultados son bastante lógicos y a la vez alentadores ya que, unidos a la tasa de preinscripción en el curso, que sobrepasó todos los años el número máximo de plazas ofertadas, pueden ser interpretados como un indicador de que las altas capacidades interesan cada vez más, los futuros profesionales son conscientes de su escasez de conocimientos, y buscan la formación necesaria para atender a estos estudiantes. Asimismo, nos permite detectar las fortalezas del curso, aquello que ha hecho que los alumnos lo elijan de entre la oferta formativa existente en la Universidad de Málaga, que parece estar relacionado con los contenidos elegidos por la dirección académica del curso y con el formato semipresencial de la estructura del curso.

En cuanto a lo que han aprendido los asistentes al curso tras seis meses de clases, hemos encontrado que los participantes han realizado aprendizajes más de tipo actitudinal que de contenidos concretos. Es decir, parece que el curso ha aumentado su sensibilidad hacia el alumnado ACIs, hacia sus familias y hacia el papel de la escuela, haciéndolos más conscientes acerca de su papel y de la necesidad de intervenir. 
Además, se observa en sus respuestas mayor accesibilidad y apertura no sólo a detectar los indicadores de alta capacidadentre los estudiantes a los que deban atender en las aulas, sino también a comprenderlos y a intervenir. Por tanto, parece que el curso los ha hecho más abiertos a aprender, a buscar recursos, a cambiar sus ideas y a actuar con sólidos conocimientos. No quiere decir esto que no hayan adquirido también ciertos aprendizaje técnicos especializados como, por ejemplo, el nombre de algunas medidas educativas (flexibilización, ampliación curricular, enriquecimiento, etc.) o algunos autores y teorías o modelos actuales explicativos de la alta capacidad, pero la presencia de estos aprendizajes más memorísticos en sus respuestas ha sido muy minoritaria.

En relación a la calidad del curso aunque la valoración general ha sido alta, parece necesario, para futuras ediciones, modificar algunos aspectos que no han resultado bien valorados como, por ejemplo, los contenidos legales o la sesión legislativa, o la coordinación de contenidos para evitar solapamientos. Asimismo, hay que mantener aquellos aspectos más valorados, como el clima del aula, creado gracias a la metodología docente empleada, o el trato cercano y empático de los profesores del curso. Además, los alumnos han valorado que permitir la entrada a alumnos de perfil muy diferente, estudiantes, profesionales, padres y madres, psicólogos de clínicas privadas, etc. haya facilitado la creación de un grupo heterogéneo con intereses comunes personales y profesionales, que no sólo han enriquecido las sesiones presenciales, sino también ha supuesto el inicio de contactos profesionales posteriores que, seguramente, redunden en una mejora de la atención educativa a los alumnos ACIs.

Nos planteamos la necesidad de evaluar el proceso de aprendizaje que se produce en cada sujeto individualmente, analizando los posibles cambios intraindividuales a lo largo de la exposición al curso, recogiendo por ejemplo información en varios momentos puntuales del ciclo formativo.Asimismo, sería interesante realizar algún análisis más pormenorizado de diferencias de medias que nos permitiera ver la relación de factores como el perfil, la titulación o las expectativas, con los procesos de aprendizaje que realizan o con la percepción del curso. Para futuros estudios sería necesario incrementar la tasa de respuesta de los asistentes, buscando formas de que más participantes cumplimenten la encuesta.

Para terminar seria conveniente decir que la propia supervivencia de cursos como este durante cinco ediciones consecutivas con tasas altas de preinscripción y matriculación es un ejemplo de la necesidad y demanda que hay en nuestra sociedad en relación a las altas capacidades. Muchos profesores, orientadores, etc. quieren formarse 
adecuadamente y buscan las vías de hacerlo. Las quejas y dificultades que denuncian los padres y madres cuando hablan de la atención educativa que se les presta a su hijos en el contexto escolar suelen ser ajustadas a lo que ocurre en muchos casos, pero también se debería valorar el esfuerzo, la motivación, el interés y las ganas de aprender de muchos otros profesionales de la escuela.

\section{Referencias}

Bernando, A. (2012). Sobre aprendices buenos y malos: la dimensión lingüística de las teorías implícitas de la inteligencia. Electronic Journal of Research in Educational Psychology, 10(1), 195-212.

Blackwell, L., Trzesniewski; K. \& Sorich Dweck, C. (2007). Implicit Theories of Intelligence Predict Achievement Across an Adolescent Transition: A Longitudinal Study and an Intervention. Child Development, 78(1), 246 - 263.

FASI (Federación de asociaciones de alta capacidad) (2011). Encuesta on line sobre la atención educativa en las escuelas. Consultado el 24 de febrero de 2015en www.fasi.es

Fernández-Molina, M. (2016). Percepción y valoración del curso de especialización en altas capacidades de la Universidad de Málaga. Datos 2011-2015. Comunicación oral personal no publicada presentada en I Congreso Internacional: Nuevas perspectivas en el estudio de la superdotación y el talento. Murcia: Universidad de Murcia.

García Bacete, F. y Rosel, J. (1999). Caracteristicas familiares y estimación de los resultados educativos de los alumnos por el profesor. Psicothema, 11(3), pp. 587600 .

\section{Como referenciar este artigo}

FERNANDEZ-MOLINA, Milagros. La formación en altas capacidades intelectuales de los profesionales psicoeducativos. Resultados del Curso de Especialización de la Universidad de Málaga. Revista on line de Política e Gestão Educacional, Araraquara, v.20, n.03, p. 668-687, 2016. Disponível em: <http://dx.doi.org/10.22633/rpge.v20.n3.9763>. ISSN: 1519-9029.

Submetido em: outubro/2016

Aprovado em: novembro/2016 